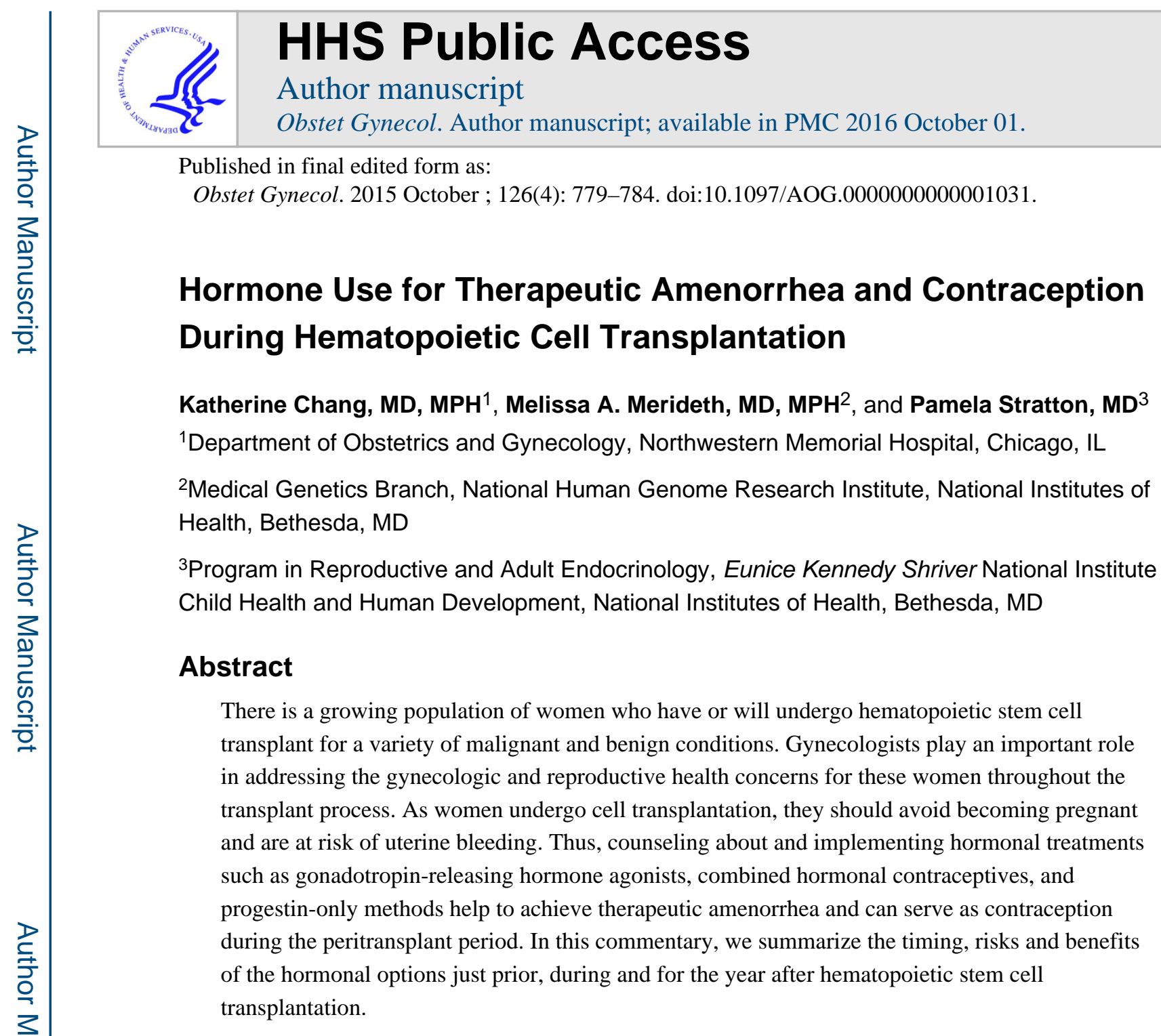

\title{
Introduction
}

Over 30 chronic and acute, malignant and nonmalignant conditions are potentially treated by hematopoietic cell transplantation with many conducted in the context of clinical investigations (Table 1). Worldwide, approximately 50,000 hematopoietic cell transplantations are performed annually, and there are an increasing number of survivors. In the United States in 2009, there were an estimated 108,900 hematopoietic cell transplant survivors, and that number is expected to increase five-fold by 2030. By that time, it is estimated that three-quarters of cell transplantation survivors will receive their transplants before or during their reproductive years.(1) As many transplant recipients are premenopausal women or girls, the team caring for cell transplant participants should include a gynecologist, along with other physicians, nurses, social workers and coordinators.

Corresponding author: Pamela Stratton, MD, Gynecology Consult Service, Program in Reproductive and Adult Endocrinology, Eunice Kennedy Shriver National Institute of Child Health and Human Development, Bldg 10, CRC, Room 1-3140, 10 Center Dr. MSC 1109, Bethesda, MD 20892-1109, Phone: 301 496-9079, Fax: 301 480-6703, ps79c@nih.gov.

Financial Disclosure: The authors did not report any potential conflicts of interest.

Presented at the American Society of Bone Marrow Transplant and Center for International Blood and Marrow Transplant Tandem Meetings on February 11th, 2015 in San Diego, California. 
The focus of this paper is on the peritransplant care of premenopausal women and their gynecologic and reproductive health considerations that directly affect long-term survival and quality of life. These considerations include prevention and treatment of heavy menstrual bleeding, fertility options, pregnancy prevention and pelvic infection prevention. Gynecologic care for post-transplant long-term survivors, including revaccination, screening for secondary cancers and chronic graft versus host disease, hormone replacement therapy, fertility, and sexuality, is discussed thoroughly elsewhere. $(2,3)$

\section{The Transplant Process}

Hematopoietic cell transplantation is undertaken with a variable amount of urgency depending on the indication for transplantation. Once a donor is identified, the recipient completes a pre-transplant evaluation and then undergoes a preparative (also known as conditioning) regimen (1-2 weeks), receives the graft infusion and then must await the initial signs of engraftment (10-28 days) and repopulation of bone marrow (60-90 days). The conditioning regimen enables the bone marrow to receive the donor cells and involves chemotherapy with or without radiation or radiolabeled monoclonal antibodies. The approach is standardized, but is tailored to the underlying disease, the patient's health status and comorbidities, and risk of rejection. However, the conditioning regimen can lead to post-transplant ovarian failure in $65-84 \%$ of patients.(4) This preparative regimen is the only gonadotoxic aspect of the transplant process, although some women may have previously received gonadotoxic chemotherapy for cancer treatment.

Conditioning regimens are classified as myeloablative, reduced intensity, and nonmyeloablative. These terms signify the effects of conditioning on the bone marrow, but practically speaking they reflect the degree of conditioning-related toxicity. Myeloablative conditioning typically results in profound pancytopenia within 1-3 weeks of the time of initiation, and if it involves total body radiation or alkylating agents, ovarian failure usually results. Non-myeloablative conditioning causes less cytopenia but significant lymphopenia initially, though engrafting donor cells eventually eliminate host cells, thus becoming myeloablative. Reduced intensity conditioning results in cytopenia and is more similar to a non-myeloablative regimen but does not fit into either of the two previous categories. In limited cases (e.g. children with severe combined immunodeficiency), transplantation may be performed without any conditioning.

Cytopenia achieved during transplantation includes thrombocytopenia, which can be problematic during menses. In general, thrombocytopenia increases the risk of heavy menstrual bleeding and may necessitate immediate transfusion and hormonal treatment. In transplant patients not receiving therapeutic menses suppression, the rate of moderate-tosevere bleeding can be as high as $40 \%$. Disease-related or iatrogenic thrombocytopenia can result in bleeding diathesis, including potentially life-threatening heavy menstrual bleeding, and may delay treatment leading to less optimal outcomes.(5)

\section{Therapeutic Amenorrhea and Family Planning}

Gynecologists play a key role in developing and initiating treatment plans to achieve the patient's individual goals. Therapeutic amenorrhea is important to prevent severe 
hemorrhage. The choice of agent depends on the time to transplant, patient's need for contraception, desire for future fertility, medical history, as well as whether emergent intervention is required in the setting of acute uterine bleeding.(6)

When emergent transplantation is not required, hormonal treatment to achieve therapeutic amenorrhea can begin prior to transplant conditioning and subsequent thrombocytopenia. Hormonal treatments can address both therapeutic amenorrhea and contraception, and possibly fertility preservation. When initiating hormonal therapy, considerations include safety, side effect profile including the risk for initial and breakthrough bleeding, duration of time before conditioning begins, and patient preference.

An overview of the hormonal treatment options for menses suppression and contraception used at our institution is found in Figure 1. These methods include gonadotropin releasing hormone agonists, combined hormonal contraceptives, and progestin-only methods, and this figure includes points to consider when discussing the options.

For achieving therapeutic amenorrhea, depot medroxyprogesterone acetate (DMPA) and gonadotropin releasing hormone agonists, are two methods that have been studied in populations undergoing cancer treatments, including the transplant population.(5) Among hematopoietic cell transplant patients, gonadotropin releasing hormone agonists are associated with high rates of amenorrhea (76.9\%).(7) In addition, $0 \%$ of gonadotropin releasing hormone agonists users experienced moderate-to-severe bleeding compared to $21.4 \%$ of DMPA users and $40 \%$ of untreated patients.(5) Gynecologists, hematologists and medical oncologists preferentially administer a gonadotropin releasing hormone agonists such as the injectable depot leuprolide acetate rather than DMPA given its increased effectiveness in achieving amenorrhea and potential for preservation of ovarian function.(2) As amenorrhea until engraftment is desired and usually occurs during the first 90 days, the preferred dose at our institution is depot leuprolide acetate $11.25 \mathrm{mg}$ intramuscularly which may be given again in three months, if needed (Figure 1).

Gonadotropin releasing hormone agonists provide both contraception and menses suppression by inducing a hypogonadotropic, hypogonadal state mimicking prepubertal (or menopausal) amenorrhea. After initiating gonadotropin releasing hormone agonists, there is an initial flare of gonadotropins and estrogen followed by down-regulation of the hypothalamic-pituitary-ovarian axis. Within the first two weeks after initial injection, withdrawal bleeding may occur. Bleeding is more likely if therapy is initiated during the follicular phase of the menstrual cycle. This therapy is most likely to suppress menses if initiated in the mid-luteal phase, one week prior to the start of the next menstrual cycle or during the use of hormonal contraception. If switching to gonadotropin releasing hormone agonists from the pill, patch or ring, continuing the short-acting method for a week or more after the injection may decrease the risk of ovarian stimulation.

When used for amenorrhea and for potential preservation of ovarian function in hematopoietic cell transplantation, gonadotropin releasing hormone agonist use is generally limited to 6 months unless specified differently in a research protocol. Side effects include hot flashes, vaginal dryness, headaches, mood changes and other menopausal symptoms 
associated with hypoestrogenism. For patients with a history of depression, there is a possibility of worsening depression and close monitoring is warranted.

Long-term hypoestrogenism is associated with progressive bone loss. For these reasons, add-back hormonal therapy with combined estrogen-progestin, progestin-only or estrogenonly agents is considered with longer use of gonadotropin releasing hormone agonists.(8) If add-back is provided via progestin-only methods, norethindrone acetate can be preferable as it has some estrogenic properties.(8) In transplant patients, bone mineral density loss is impossible to attribute to gonadotropin releasing hormone agonist therapy alone given the concurrent use of steroids or other medications affecting bone metabolism and the hypoestrogenic state from ovarian dysfunction post-transplant.(9)

Studies indicate that side effects often associated with gonadotropin releasing hormone agonist use are not problematic in transplant patients.(2) This method also does not have the prothrombotic effects seen in other hormonal agents. In addition, the potential of preserving ovarian function by using gonadotropin releasing hormone agonists offers an additional reproductive health benefit; the postulated mechanisms include decreasing utero-ovarian perfusion, protecting undifferentiated germ-line stem cells, or up-regulation of intragonadal antiapoptotic molecules such as sphingosine-1-phosphate.(3) Some studies demonstrate concurrent gonadotropin releasing hormone agonist administration with gonadotoxic chemotherapy decreases the rate of premature ovarian failure and increases the probability of future pregnancy, $(9,10)$ particularly in breast cancer patients.(11) However, at this time studies remain inconclusive on the matter according to a recent meta-analysis.(12) More investigation, including randomized controlled trials particularly in transplant patients, is needed.

Menses suppression is continued for at least 90 days post-transplant until engraftment occurs and thrombocytopenia resolves (Figure 1). Transient post-transplant ovarian dysfunction can be common in the first two years after transplant,(13) even in patients who eventually experience a return of normal, cyclic menses. In those undergoing transplant for cancer treatment, the risk of relapse is greatest during this time.

Pregnancy during cell transplantation is contraindicated because of the potential teratogenicity of the conditioning treatment. Pregnancy should also be avoided during the first two years post-transplant due to the risk of relapse, and management of other posttransplant complications. Therefore, use of reliable contraceptive methods is important during the first two years post-transplant. Patients are advised to use contraception even though their sexual activity may be limited. Disease, treatment-related symptoms, medications, and stress may each negatively affect sexuality and libido.

Long-acting reversible contraceptive (LARC) methods such as intrauterine devices (IUDs) or implants are highly effective in avoiding unplanned pregnancies, but have not been well studied in the transplant population. Recommendations regarding continuation of LARC methods may differ by device characteristics.

The etonorgestrel single-rod implant can provide menses suppression and may be continued if this has occurred. While copper IUDs are highly effective as contraceptives, they neither 
suppress menses nor decrease the risk of infection and they should be removed prior to initiation of the conditioning regimen.

In contrast, the levonorgestrel IUDs are a not only highly effective contraceptives, but may suppress menses and are associated with a lower risk of pelvic infection. For transplant patients, infection during neutropenia or active immunosuppression may occur with an intrauterine foreign body. No studies, albeit limited, suggest an increased likelihood of febrile morbidity with progestin-containing IUD use in immunosuppressed women.(6) In the absence of data regarding safety and efficacy, if a patient is already amenorrheic with a progestin-containing IUD or implant, its continued use while undergoing hematopoietic cell transplantation may be considered.(14) The patient is then counseled that in the event of neutropenic fever arising during or after transplant, a request to remove the progestincontaining IUD may occur. Ultimately, the transplant team, gynecology team and patient decide together whether to keep the IUD in place or remove it prior to transplant, transitioning to an alternate method of menses suppression and contraception. Due to an unpredictable bleeding profile immediately post-insertion of IUDs and implants, these methods are not initiated as part of the transplant care as they do not lead to therapeutic amenorrhea prior to thrombocytopenia.

Women may be using shorter-acting hormonal methods like a pill, patch, ring, or injection. If a woman already has amenorrhea with DMPA use at the time of transplant, it may be continued. Combined hormonal therapies may confer an increased risk of veno-occlusive disease during transplant due to the estrogen component and third-generation progestins in some.(15) Women with an increased risk of thromboembolic events, such as pre-existing thrombophilias, disease-related increased risk, or myeloablative conditioning generally are not prescribed estrogen-containing therapies. The incidence of venous thromboembolism (DVT) in patients undergoing transplant for malignancies is $3.4-4.6 \%$, and is associated with longer hospitalization, prior DVT, graft versus host disease and corticosteroid use. $(16,17)$ Whether this incidence and the characteristics conferring risk is similar in patients undergoing transplant for non-malignant indications or with reduced-intensity conditioning is not known.

Progestin-only methods such as norethindrone acetate or medroxyprogesterone acetate can effectively provide menses suppression and may be preferable if there are contraindications to estrogen use (Figure 1). Treatment-related nausea, vomiting or mucositis can worsen tolerability and absorption of oral medications, requiring a change in administration route. Similarly, skin sensitivities or graft versus host disease may preclude the use of transdermal patches. Most would recommend discontinuing vaginal rings prior to transplant given the theoretical risk of infection arising from a foreign body in an unsterile cavity during pancytopenia.

Counseling on future fertility and options for potential fertility preservation are addressed as part of the pre-transplant evaluation. Fertility preservation options include a combination of in vitro fertilization and ovarian, embryo, or oocyte cryopreservation. For those receiving radiation therapy, ovarian shielding or transposition can also be performed. Expeditious referral to a reproductive endocrinologist and infertility specialist is warranted for those who 
desire use of assisted reproductive technologies to enable cryopreservation of gametes, but would not be feasible for the patient requiring urgent transplantation and immediate initiation of chemotherapy.

These procedures can be performed during the time used for transplant preparations such as identifying a donor. If ovulation induction is performed, considerations are taken to avoid precipitating further ovarian stimulation when achieving therapeutic amenorrhea, such as the use of gonadotropin releasing hormone antagonists after oocyte retrieval. Use of gonadotropin releasing hormone agonists as a fertility preservation option is being studied and is considered investigational. The details of counseling on fertility preservation options are reviewed in detail elsewhere. $(4,18)$

\section{Treatment of heavy menstrual bleeding}

If a hemodynamically stable patient presents with heavy menstrual bleeding and there are no contraindications to estrogen use, continuous monophasic OCP containing 30-35 micrograms of ethinyl estradiol can be used for initial treatment along with blood product transfusions.(19) If bleeding does not subside, the dose can be doubled to one pill twice daily.(19) In severe cases, patients can receive intravenous conjugated equine estrogen (25 $\mathrm{mg}$ every 6 hours for 24 hours). If a patient has contraindications to estrogen use, gonadotropin-releasing hormone agonists or continuous progestins are treatment options. (20) Oral progestin (medroxyprogesterone acetate $20 \mathrm{mg}$ three times daily for one week, then once daily for the next three weeks) was equally effective and tolerated in treating hemodynamically stable acute uterine bleeding when compared with combined OCP containing 35 micrograms of ethinyl estradiol in one small randomized control trial.(21) If bleeding continues or the clinical status necessitates more immediate intervention, options include intrauterine balloon tamponade, antifibrinolytics, curettage, uterine artery embolization, endometrial ablation, or hysterectomy.(19)

\section{Reproductive health concerns immediately after cell transplantation}

During the first year post-transplant, transitioning to estrogen-containing therapy provides contraception, menses suppression, menopausal symptoms and hormone replacement to reduce bone mineral density loss, particularly in younger patients. For example, patients who use gonadotropin releasing hormone agonists during transplant could transition to combined OCPs after engraftment or thrombocytopenia resolves if there are no contraindications and the patient is medically stable. Engraftment often occurs by 3 to 6 months post-transplant.

\section{Limitations and future directions}

As the number of indications for hematopoietic cell transplantations is growing, interest and research in management of related gynecologic issues related will increase. Studies are needed on LARC methods in transplant patients, as well randomized trials assessing the efficacy of gonadotropin releasing hormone agonists in preserving ovarian function. A timely gynecology referral and a multidisciplinary team approach can address various issues in pre-menopausal transplant patients, including counseling on hormonal treatment options. 
Thus, in order to serve as a valuable consultant, it is important for the obstetriciangynecologist to be aware of the unique concerns that may arise for transplant patients and recent advances in this field. At our institution, multidisciplinary care teams are a critical, vital aspect of our institutional culture. As such, as seen in Figure 1, gynecologic and reproductive health issues are considered an integral part of a pre-transplant assessment. By partnering with our transplant colleagues, gynecologists create an integrated approach to care for these women enabling us to consider and address both common and unique women's health issues in the context of transplant.

\section{Acknowledgments}

Funded by the Intramural Program of the NIH, Clinical Center, the Eunice Kennedy Shriver National Institute of Child Health and Human Development, and the National Human Genome Research Institute.

The authors thank the Intramural Program hematopoietic transplant teams at the National Heart, Lung, and Blood Institute, National Cancer Institute, National Institute of Allergy and Infectious Diseases and National Institute of Diabetes, Digestive, and Kidney Diseases for their collaboration during the transplantation of female patients and to the female transplant patients themselves for their interest in their gynecologic and reproductive health.

\section{References}

1. Majhail NS, Tao L, Bredeson C, Davies S, Dehn J, Gajewski JL, et al. Prevalence of hematopoietic cell transplant survivors in the United States. Biology of blood and marrow transplantation : journal of the American Society for Blood and Marrow Transplantation. 2013 Oct; 19(10):1498-501.

2. Milroy CL, Jones KP. Gynecologic care in hematopoietic stem cell transplant patients: a review. Obstetrical \& gynecological survey. 2010 Oct; 65(10):668-79. [PubMed: 21182805]

3. Shanis D, Merideth M, Pulanic TK, Savani BN, Battiwalla M, Stratton P. Female long-term survivors after allogeneic hematopoietic stem cell transplantation: evaluation and management. Seminars in hematology. 2012 Jan; 49(1):83-93. [PubMed: 22221788]

4. Joshi S, Savani BN, Chow EJ, Gilleece MH, Halter J, Jacobsohn DA, et al. Clinical guide to fertility preservation in hematopoietic cell transplant recipients. Bone marrow transplantation. $2014 \mathrm{Apr}$; 49(4):477-84. [PubMed: 24419521]

5. Meirow D, Rabinovici J, Katz D, Or R, Shufaro Y, Ben-Yehuda D. Prevention of severe menorrhagia in oncology patients with treatment-induced thrombocytopenia by luteinizing hormone-releasing hormone agonist and depo-medroxyprogesterone acetate. Cancer. 2006 Oct 1; 107(7):1634-41. [PubMed: 16944540]

6. Martin-Johnston MK, Okoji OY, Armstrong A. Therapeutic amenorrhea in patients at risk for thrombocytopenia. Obstetrical \& gynecological survey. 2008 Jun; 63(6):395-402. quiz 5. [PubMed: 18492296]

7. Quaas AM, Ginsburg ES. Prevention and treatment of uterine bleeding in hematologic malignancy. European Journal of Obstetrics Gynecology and Reproductive Biology. 2007 Sep; 134(1):3-8.

8. Chwalisz K, Surrey E, Stanczyk FZ. The Hormonal Profile of Norethindrone Acetate: Rationale for Add-Back Therapy With Gonadotropin-Releasing Hormone Agonists in Women With Endometriosis. Reproductive Sciences. 2012 Jun; 19(6):563-71. [PubMed: 22457429]

9. Del Mastro L, Boni L, Michelotti A, Gamucci T, Olmeo N, Gori S, et al. Effect of the gonadotropinreleasing hormone analogue triptorelin on the occurrence of chemotherapy-induced early menopause in premenopausal women with breast cancer: a randomized trial. Jama. 2011 Jul 20; 306(3):269-76. [PubMed: 21771987]

10. Blumenfeld Z, Patel B, Leiba R, Zuckerman T. Gonadotropin-releasing hormone agonist may minimize premature ovarian failure in young women undergoing autologous stem cell transplantation. Fertility and sterility. 2012 Nov; 98(5):1266-70. e1. [PubMed: 22935556] 
11. Moore HC, Unger JM, Phillips KA, Boyle F, Hitre E, Porter D, et al. Goserelin for ovarian protection during breast-cancer adjuvant chemotherapy. The New England journal of medicine. 2015 Mar 5; 372(10):923-32. [PubMed: 25738668]

12. Kim SS, Lee JR, Jee BC, Suh CS, Kim SH, Ting A, et al. Use of hormonal protection for chemotherapy-induced gonadotoxicity. Clinical obstetrics and gynecology. 2010 Dec; 53(4):74052. [PubMed: 21048441]

13. Liu J, Malhotra R, Voltarelli J, Stracieri AB, Oliveira L, Simoes BP, et al. Ovarian recovery after stem cell transplantation. Bone marrow transplantation. 2008 Feb; 41(3):275-8. [PubMed: 17952128]

14. Committee opinion no. 606: Options for prevention and management of heavy menstrual bleeding in adolescent patients undergoing cancer treatment. Obstetrics and gynecology. 2014 Aug; 124(2 Pt 1):397-402. [PubMed: 25050771]

15. Kiley J, Hammond C. Combined oral contraceptives: a comprehensive review. Clinical obstetrics and gynecology. 2007 Dec; 50(4):868-77. [PubMed: 17982329]

16. Gerber DE, Segal JB, Levy MY, Kane J, Jones RJ, Streiff MB. The incidence of and risk factors for venous thromboembolism (VTE) and bleeding among 1514 patients undergoing hematopoietic stem cell transplantation: implications for VTE prevention. Blood. 2008 Aug 1; 112(3):504-10. [PubMed: 18480425]

17. Gonsalves A, Carrier M, Wells PS, McDiarmid SA, Huebsch LB, Allan DS. Incidence of symptomatic venous thromboembolism following hematopoietic stem cell transplantation. Journal of thrombosis and haemostasis : JTH. 2008 Sep; 6(9):1468-73. [PubMed: 18627443]

18. Practice Committee of American Society for Reproductive M. Fertility preservation in patients undergoing gonadotoxic therapy or gonadectomy: a committee opinion. Fertility and sterility. 2013 Nov; 100(5):1214-23. [PubMed: 24011612]

19. Levens ED, Scheinberg P, DeCherney AH. Severe menorrhagia associated with thrombocytopenia. Obstetrics and gynecology. 2007 Oct; 110(4):913-7. [PubMed: 17906028]

20. Singh S, Best C, Dunn S, Leyland N, Wolfman WL, et al. Clinical Practice - Gynaecology C. Abnormal uterine bleeding in pre-menopausal women. Journal of obstetrics and gynaecology Canada : JOGC = Journal d'obstetrique et gynecologie du Canada : JOGC. 2013 May; 35(5):4739.

21. Munro MG, Mainor N, Basu R, Brisinger M, Barreda L. Oral medroxyprogesterone acetate and combination oral contraceptives for acute uterine bleeding: a randomized controlled trial. Obstetrics and gynecology. 2006 Oct; 108(4):924-9. [PubMed: 17012455] 


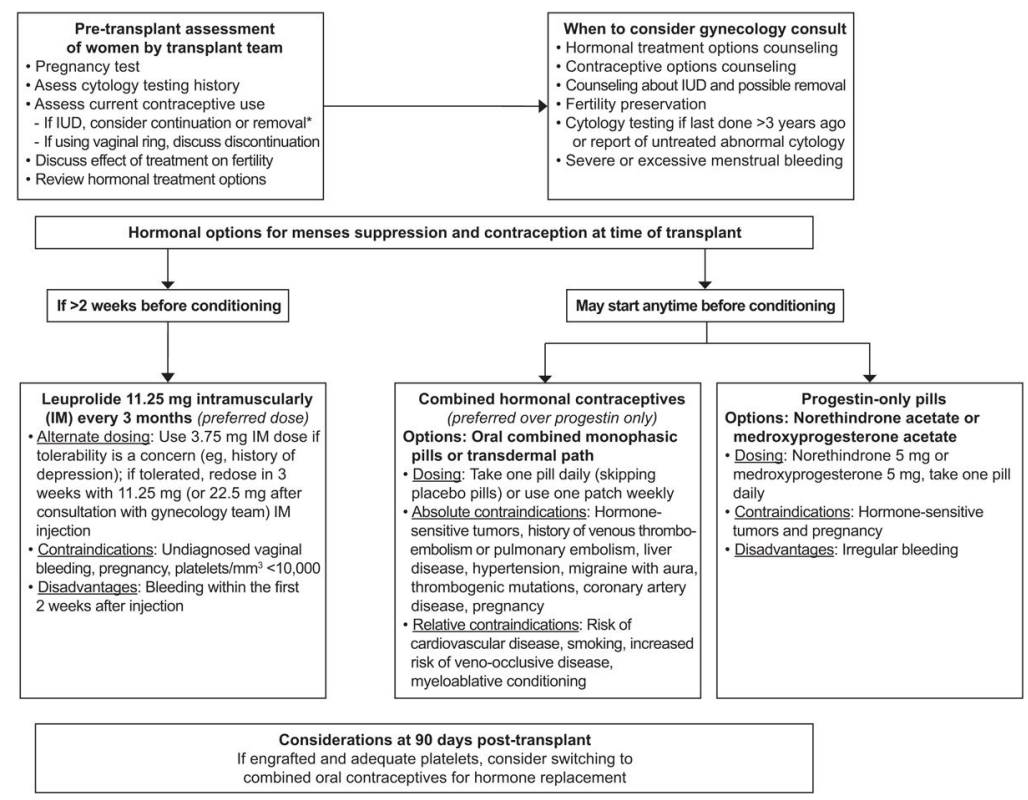

Figure 1.

Hormonal treatment options for therapeutic amenorrhea and contraception. IUD, intrauterine device. *Copper IUDs should be removed. Levonorgesterel IUDs may be continued or removed after discussion between the patient, transplant team, and gynecology team. The patient can be counseled that removal may be requested if she develops neutropenic fever during transplant. 
Table 1

Conditions treated with hematopoietic stem cell transplants

\begin{tabular}{|c|c|}
\hline Allogeneic stem cell transplants & Autologous stem cell transplants \\
\hline Hematologic malignancies & Hematologic malignancies \\
\hline Acute myeloid leukemia & Multiple myeloma \\
\hline Acute lymphoblastic leukemia & Hodgkin lymphoma \\
\hline Chronic lymphocytic leukemia & Follicular lymphoma \\
\hline Chronic myeloid leukemia & Diffuse large B cell lymphoma \\
\hline Peripheral T cell lymphoma & Peripheral T cell lymphoma \\
\hline Follicular lymphoma & Mantle cell lymphoma \\
\hline Diffuse large B cell lymphoma & Acute myeloid leukemia \\
\hline Multiple myeloma* (in clinical trials) & Waldenström macroglbulinemia \\
\hline Nonhematologic malignancies & Other \\
\hline Neuroblastoma & Immunoglobulin light chain amyloidosis \\
\hline \multicolumn{2}{|c|}{ Nonmalignant inherited or acquired marrow disorders } \\
\hline \multicolumn{2}{|l|}{ Sickle cell anemia } \\
\hline \multicolumn{2}{|l|}{ Beta cell thalassemia major } \\
\hline \multicolumn{2}{|l|}{ Refractory Diamond-Blackfan anemia } \\
\hline \multicolumn{2}{|l|}{ Myelodysplastic syndrome } \\
\hline \multicolumn{2}{|l|}{ Idiopathic severe aplastic anemia } \\
\hline \multicolumn{2}{|l|}{ Paroxysmal nocturnal hemoglobinuria } \\
\hline \multicolumn{2}{|l|}{ Pure red cell aplasia } \\
\hline \multicolumn{2}{|l|}{ Fanconi anemia } \\
\hline \multicolumn{2}{|l|}{ Dyskeratosis Congenita } \\
\hline \multicolumn{2}{|l|}{ Amegakaryocytosis } \\
\hline \multicolumn{2}{|l|}{ Congenital thrombocytopenia } \\
\hline \multicolumn{2}{|c|}{ Autoimmune disease, inborn errors in metabolism, or congenital immune deficiencies } \\
\hline \multicolumn{2}{|l|}{ Primary immunodeficiency } \\
\hline \multicolumn{2}{|l|}{ Adrenoleukodystrophy } \\
\hline \multicolumn{2}{|l|}{ Mucopolysacharidoses } \\
\hline \multicolumn{2}{|l|}{ Severe combined immunodeficiency } \\
\hline \multicolumn{2}{|l|}{ Chronic granulomatous disease } \\
\hline \multicolumn{2}{|l|}{ Krabbe disease } \\
\hline \multicolumn{2}{|l|}{ NK cell deficiency syndromes } \\
\hline \multicolumn{2}{|l|}{ DiGeorge syndrome } \\
\hline \multicolumn{2}{|l|}{ Wiskott-Aldrich syndrome } \\
\hline \multicolumn{2}{|l|}{ GATA2 haploinsufficiency } \\
\hline \multicolumn{2}{|l|}{ DOCK-8 deficiency } \\
\hline Osteopetrosis & \\
\hline
\end{tabular}

